\title{
Téoros
}

Revue de recherche en tourisme

\section{Tourisme identitaire et spécialisation infra-régionale dans les petites économies isolées}

\section{Denis Serra}

Volume 19, numéro 3, automne 2000

Organisations touristiques en mutation

URI : https://id.erudit.org/iderudit/1071738ar

DOI : https://doi.org/10.7202/1071738ar

Aller au sommaire du numéro

Éditeur(s)

Université du Québec à Montréal

ISSN

0712-8657 (imprimé)

1923-2705 (numérique)

Découvrir la revue

Citer cet article

Serra, D. (2000). Tourisme identitaire et spécialisation infra-régionale dans les petites économies isolées. Téoros, 19(3), 20-27.

https://doi.org/10.7202/1071738ar d'utilisation que vous pouvez consulter en ligne. 


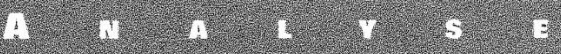 \\ TOURISME IDENTITAIRE ET SPÉCIALISATION INFRA-RÉGIONALE DANS LES PETITES ÉCONOMIES ISOLÉES}

\section{Denis Serra}

Le tourisme est souvent l'activité économique principale des petites économies isolées, en particulier dans les milieux insulaires. Pourtant, trop souvent concentrée dans le temps (saison estivale) et dans l'espace (littoral), l'activité touristique a un effet nécessairement limité sur l'économie régionale et ne suffit pas à donner à ces régions l'élan qui leur manque pour sortir d'un état de «mal-développement». Fondamentalement, cette concentration spatio-temporelle se traduit par une faible diversification de l'offre touristique qui, dans un contexte concurrentiel, est un lourd handicap que les régions doivent surmonter pour réaliser leur développement.

Après avoir mis en évidence les problèmes liés à la petite taille et à l'isolement de ces régions, nous montrerons que le tourisme et plus précisément la mise en valeur des spécificités intrinsèques, « identitaires ${ }^{1} \gg$ de ces régions, peuvent conduire à un essor certain de leur économie.

\section{DIFFICULTÉS LIÉES À L'INSULARITÉ}

Les analyses récentes d'économie régionale et notamment le courant de l'économie géographique semblent ne donner aucune chance à la survie des petites économies isolées. En effet, la baisse des coûts de transport conduit à une intensification de la concurrence par les prix et amène les entreprises à diversifier leur production pour reconstituer leur pouvoir sur le marché. La diversification de la production nécessite une différenciation du travail qui n'est possible que si le marché du travail a une taille suffisante (pour qu'il puisse être segmenté comme dans les grandes agglomérations). Dans les grandes agglomérations, on incite les entreprises se regrouper pour profiter de la proximité de la demande. Les consommateurs ont, pour leur part, intérêt à s'installer près d'entreprises qui offrent une gamme variée de produits. Ce processus de « polarisation » des régions de grande taille possède un caractère cumulatif alimenté non seulement par l'interaction « entreprises / salariés », mais également par l'interaction « entreprises / consommateurs ». Ces interactions sont à la base d'une véfait aux dépens des régions isolées de petite taille, qui n'ont pas la capacité de répondre à l'intensification de la concurrence par les prix grâce à la différenciation des produits locaux. Le cas de la Corse illustre parfaitement cette analyse : la baisse des coûts de transport engendrée par la navigation à vapeur au XIX ${ }^{\mathrm{e}}$ siècle a conduit à un déclin de l'économie régionale et à une dépendance de l'île par rapport aux régions continentales.

\section{DIfFICULTÉS LIÉES À LA PETITE TAILLE DU MARCHÉ}

Sur un petit territoire, il est difficile de bénéficier d'économies d'échelle. Rappelons qu'il y a économie d'échelle quand l'augmentation des quantités produites se ritable dynamique de polarisation qui se traduit par une réduction du coût unitaire. On parle également de rendements d'échelle croissants. Le rendement est la relation entre les variations des quantités produites (output) et les variations des facteurs nécessaires pour les produire (input). Les rendements d'échelle relient la production à une combinaison de facteurs qui varient tous deux simultanément ; ils sont croissants si l'output augmente dans une proportion plus grande. Il y a alors économie d'échelle, l'augmentation de l'échelle de la production permettant de réduire le coût par unité produite (graphique 1) (Brémond et Gélédan, 1984).

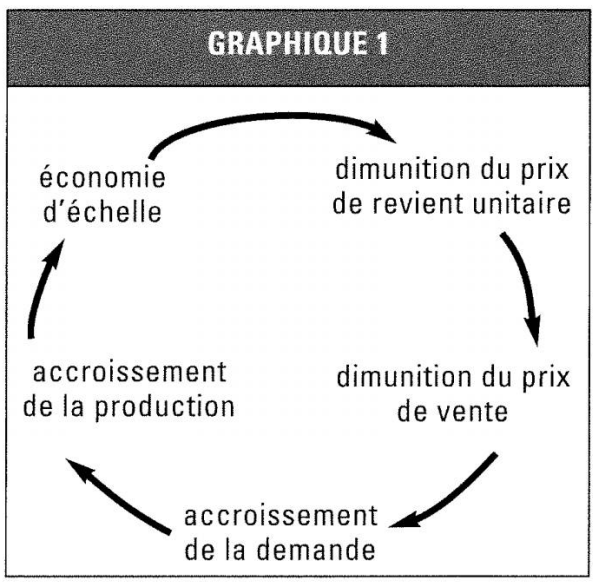

Dans de nombreux domaines, le progrès technologique a été marqué par l'invention de machines permettant d'accroître la productivité. Le plus souvent, ce matériel n'est rentable que si la production réalisée est importante. L'exemple le plus cité est sans doute celui de la chaîne continue automobile, mise en place par Ford pour produire les « Ford $\mathrm{T}$ », qui permettait de réduire le prix de vente unitaire dans des proportions impressionnantes, mais qui n'était rentable que si la voiture devenait 
un objet populaire. Une production de masse pour une consommation de masse devait ainsi permettre une extension considérable et « auto-entretenue » de la production et de la consommation. Bénéficier de ces économies d'échelle a été l'un des objectifs de la forte concentration qui a marqué le secteur automobile dans tous les pays industrialisés depuis le début du siècle. Dans tous les secteurs ou dans tous les systèmes productifs où les coûts fixes sont importants, les possibilités d'économie d'échelle sont fortes.

On comprend alors le problème qui peut se poser au niveau des petites économies isolées. Leur petite dimension va représenter une contrainte importante au processus de développement. En effet, toute production destinée au marché intérieur sera réduite puisque l'économie, donc le marché et aussi la demande, se limitent à la population. Dans ces conditions, il sera difficile de bénéficier d'économies d'échelle puisque l'insuffisance de structures démographiques, urbaines et commerciales empêche une répartition harmonieuse de l'amortissement des infrastructures nécessaires au développement touristique sur une production suffisamment diversifiée. Il en résulte des surcoûts qui pèsent sur la compétitivité de la plupart des produits touristiques du fait du prix particulièrement élevé de l'électricité, de l'eau potable et des produits importés (à cause du coût des transports) $)^{2}$. Ces contraintes sont particulièrement importantes quand les flux touristiques sont faibles comme c'est le cas pour un grand nombre d'îles du Pacifique.

Cependant, dans le cadre du commerce international - donc non plus sur le marché intérieur mais dans le contexte d'une économie mondiale intégrée grâce aux échanges commerciaux -, une économie même petite qui, de plus, saurait tirer profit du processus de spécialisation internationale, pourrait bénéficier du phénomène d'économie d'échelle dans ses domaines de «prédilection». Il serait autrement difficile de comprendre le cas d'économies comme celle de Singapour qui a réussi à acquérir un niveau de concurrence internationale non grâce à sa taille, mais plutôt en exploitant les occasions que le marché mondial lui offrait. Dans le cas d'économies d'échelle internationales, il est évident que la dimension réduite ne pose aucune contrainte à la croissance. Rappelons que nous nous intéressons, pour notre part, à des économies de taille réduite, mais aussi « isolées ».

\section{DIFFICULTÉS LIÉES À L'ISOLEMENT}

Une île qui aurait la possibilité de proposer un produit «identitaire » ou pour lequel elle aurait une facilité de production exceptionnelle lui conférant une situation de quasi-monopole pourrait bénéficier d'une économie d'échelle quasi illimitée puisque le marché (de la demande) serait luimême quasi illimité (du fait de la rareté ou du tarif préférentiel pratiqué par l'île disposant d'une facilité de production particulière pour ce produit). Se posera alors le problème de l'approvisionnement du marché. Si le coût moyen de production est en baisse constante (effet de l'économie d'échelle quasi illimitée), les coûts de transport pour irriguer le marché augmentent à mesure que l'on s'éloigne du lieu de fabrication, ce qui provoquera à un moment donné (fonction de la distance d'acheminement du produit) une augmentation du coût total par unité (transport compris). Le graphique 2 permet de visualiser nos remarques.

L'augmentation de la production permettant une diminution constante du coût par unité (et donc du coût moyen), il y a économie d'échelle, à condition de pouvoir distiller la production sur le marché. L'île devra donc satisfaire une demande de plus en plus éloignée pour distribuer son produit et donc intégrer au coût de revient des frais d'acheminement de plus en plus lourds, même si l'on considère que les coûts de transport ne forment pas, en réalité, une fonction linéaire mais, après une forte augmentation initiale, qu'ils ont tendance à croître plus faiblement quand une longue distance est atteinte ${ }^{3}$. Ainsi, pour une quantité de produit supérieure à Q2, le coût supplémentaire d'acheminement sur le marché un peu plus éloigné sera plus important que l'économie qu'aura engendrée sa production. Ce raisonnement est généralement mis de l'avant pour expliquer le phénomène de «centralité » en économie régionale ou spatiale (Polèse, 1994).

On peut en conclure que, même s'il peut exister un phénomène de rendements croissants pour les petites économies (par le biais d'un marché extérieur important) et même si le fait d'être isolée n'empêche pas une région d'avoir des activités économiques, le cumul de ces deux propriétés impose parfois quelques limites au développement (ou du moins à la croissance) de ces petites économies isolées.

\section{LA SPÉCIALISATION " INFRA-RÉGIONALE "}

Les petites économies isolées désireuses de se sortir d'une éventuelle situation de stagnation économique n'ont d'autres choix que celui de tout mettre en œuvre pour participer au commerce international. Être présent sur le marché international devient pour elles un enjeu majeur. Pour

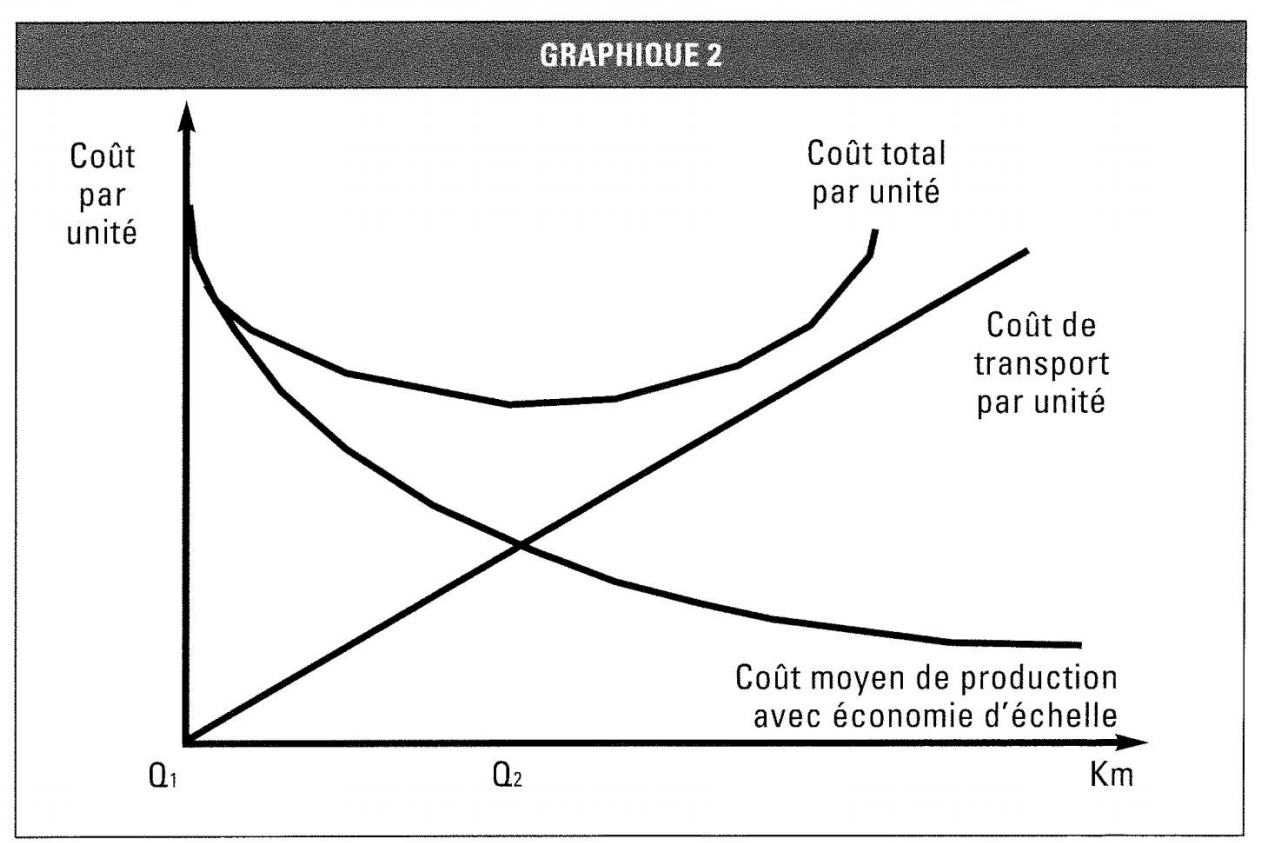


réaliser cela, les principes de spécialisation internationale leur confèrent des opportunités saisissables, pour peu qu'elles parviennent à se mettre en valeur en proposant des produits de qualité.

Les petites économies insulaires, disposant d'atouts principalement relatifs à leur forte dotation en ressources naturelles, auront souvent intérêt à se spécialiser dans le tourisme. Evidemment, il ne suffit pas de choisir de se spécialiser pour y parvenir et récolter les bénéfices attendus. C'est tout un plan, une stratégie de développement, qui doit être élaboré, et cela en fonction des particularités du milieu, de ses spécificités. Il faudra notamment :

- Mettre en valeur les produits «identitaires » susceptibles de procurer à la région un avantage absolu par rapport à l'offre touristique internationale (puisque, nous le verrons, un produit identitaire procure à son détenteur une situation de monopole sur ce bien).

- Rendre le plus efficient possible les produits « classiques »; les entretenir, les améliorer en se tenant au courant des nouvelles technologies pour bénéficier également de la demande issue de pays voisins (et semblables).

- Mais aussi, et surtout, ne pas tout miser sur le seul secteur de base que deviendrait alors le tourisme dans l'île et mettre en place une véritable politique de «participation » des secteurs résiduels (résidentiels) ${ }^{4}$ - comme l'agriculture, l'élevage, l'artisanat et toute forme de service (non directement lié au tourisme) - qui pourraient devenir les principaux fournisseurs et donc, en quelque sorte, le moteur de l'activité de base (il ne faut, en effet, jamais perdre de vue qu'en ce qui concerne le tourisme, rien ne peut se faire sans l'accord de la population locale).

Les enseignements de la théorie du commerce international nous amènent à une spécialisation dans l'activité touristique des petites régions isolées. Mais pour se conformer aux enseignements de la géographie économique, cette spécialisation doit être compatible avec une différenciation des produits touristiques offerts (la baisse des coûts de transport accroît la concurrence entre les différentes destinations et nécessite plus que jamais une diversification de l'activité touristique de ces dernières). Pour concilier spécialisation et diversification, pour envisager une spécialisation par la diversification, il faut appréhender une région comme étant en fait un regroupement de plusieurs entités et, à partir de là, appliquer la théorie des avantages comparatifs de Ricardo à chaque composante de la région. Alors, la diversification régionale du produit touristique résultera de la spécialisation infra-régionale.

On démontre l'intérêt de cette forme de spécialisation en prenant l'exemple simple de l'implantation de deux types d'établissements qui représentent en fait deux types de produits touristiques différents : un hôtel et un camping, dans deux zones disposant également de caractéristiques différentes :

- Une zone « verte » (V) d'aspect relativement sensible et faiblement confortable. D'un aspect pittoresque certain, pas forcément très accessible, mais pas isolée non plus sa faune et sa flore ont été jusqu'ici entretenues et protégées. Elle n'est pas excessivement développée commercialement, on y trouve quelques commerces sans luxe : plutôt pizzeria que restaurant avec étoiles, plutôt cinéma de plein air que casino. Elle ne dispose ni d'une grande capacité d'accueil, ni d'une forte attraction touristique ; si ce n'est par son aspect proche de la nature.

- Une zone de «luxe» (L) d'aspect relativement confortable et faiblement sensible. Elle dispose d'attractions touristiques, d'activités commerciales no-

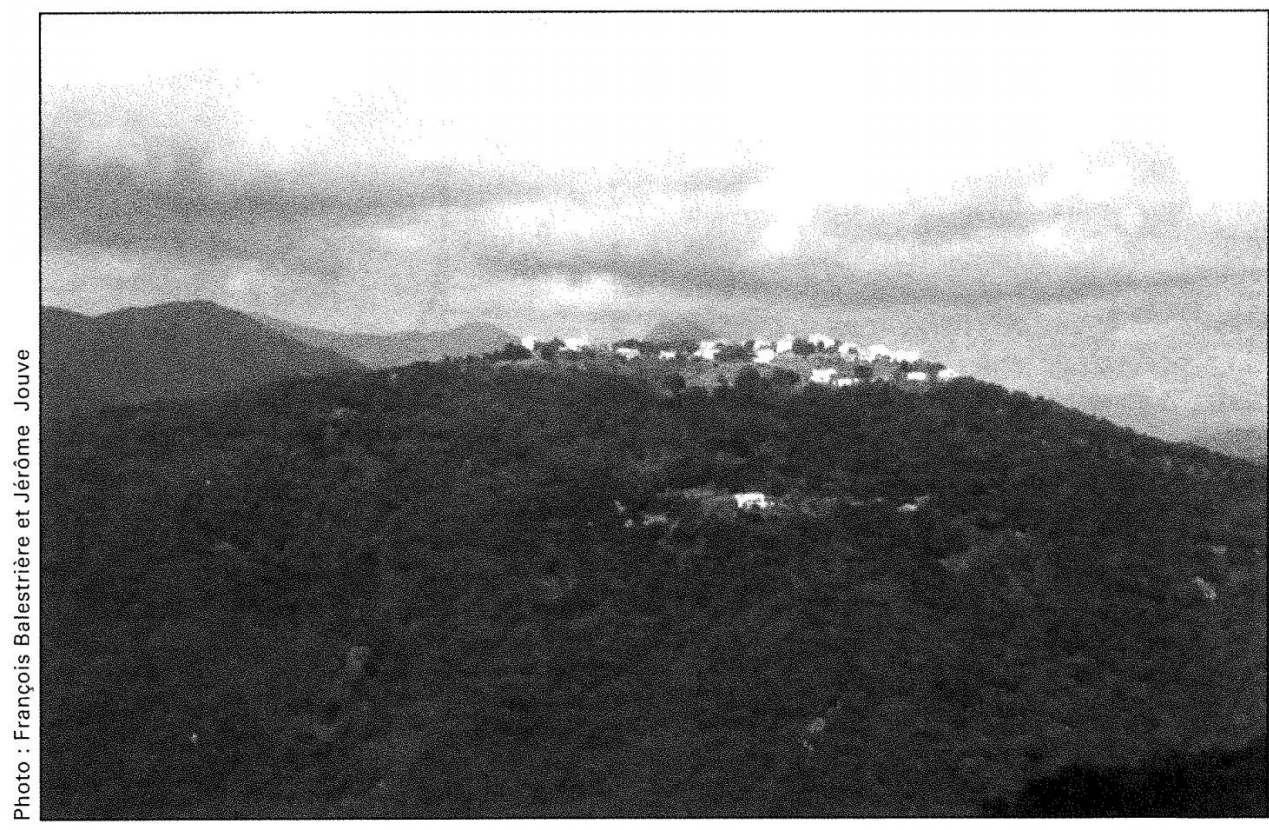

Village de Fiumorbo
TABIEAU 1

Flux monétaires, en Unite Monátaire (UM). engendres par $\mathrm{H}$ et C sur Lou V

\begin{tabular}{|l|c|c|}
\hline $\begin{array}{l}\text { Flux } \\
\text { monétaires }\end{array}$ & Zone de luxe & Zone verte \\
\hline Hôtel & 500 & 200 \\
\hline Camping & 200 & 150 \\
\hline
\end{tabular}

tables et d'une capacité d'accueil relativement importante. De plus, elle est facilement accessible. Finalement, elle ne dispose ni d'un patrimoine historique ni d'une faune et d'une flore remarquables.

Dans ces conditions et pour donner à notre modèle des valeurs chiffrées, les flux monétaires susceptibles d'être engendrés par un camping et un hôtel implantés sur L ou V sont représentés au tableau 1 (on peut considérer cela comme une moyenne représentative) :

On note alors que :

- Où qu'il soit implanté, l'hôtel engendre un flux monétaire supérieur à celui engendré par le camping : en L : $500>200$; en $\mathrm{V}: 200>150$.

- Quel que soit l'établissement considéré, il engendrera un flux monétaire plus 


\begin{tabular}{|l|c|c|c|}
\hline \multicolumn{2}{|c|}{ TABLEAU 2 } \\
\hline Calcul des flux monétaires engendres \\
\hline Elux monétaires & Zone de " luxe " & Zone " verte " & Macro-zone VL \\
\hline Deux campings & $2200=400$ & $2150=300$ & 700 \\
\hline Gains & 900 & 500 & 1400 \\
\hline
\end{tabular}

\begin{tabular}{|l|c|c|}
\hline & $\begin{array}{c}\text { TABLEAU 3 } \\
\text { Caloul des flux relatifs }\end{array}$ & Zone " verte " \\
\hline Flux relatifs & Zone de " luxe " & $200 / 150=1,33$ \\
\hline Hôtel / Camping & $500 / 200=2,5$ & $150 / 200=0,75$ \\
\hline Camping / Hôtel & $200 / 500=0,4$ & \\
\hline
\end{tabular}

important dans une zone de «luxe » que dans une zone «verte». Pour $\mathrm{H}$ : $500>200$; pour C : $200>150$.

Supposons alors qu'une subvention soit accordée à la macro-zone V-L afin de faciliter son développement touristique par l'implantation de six établissements d'accueil. Par souci de décentralisation des pouvoirs et d'une certaine autonomie accordée au niveau local (à la macro-zone) quant à la prise en main de son développement, aucune condition relative au type d'établissement à implanter ou au lieu d'implantation n'est émise par l'instance bienfaitrice. Les décideurs des zones verte et de luxe, respectivement DV et DL, se mettent rapidement d'accord pour respecter la demande touristique sur la macrozone qui est, par exemple, deux fois plus

importante en camping qu'en hôtel. Deux hôtels et quatre campings seront donc implantés sur la macro-zone. DV et DL proposent « logiquement » de se partager équitablement les établissements à mettre en place. Chacun d'eux aura donc pour tâche d'exploiter un hôtel et deux campings.

Ils s'empressent alors d'estimer les flux monétaires supplémentaires que leur fourniront ces trois nouveaux établissements, puisqu'ils connaissent les évaluations (moyennes) présentées au tableau précédent. Le tableau 2 présente le calcul des deux décideurs.

Si les deux décideurs s'étaient interrogés sur une possible application de la théorie des avantages comparatifs de Ricardo, ils

\begin{tabular}{|l|c|c|c|}
\hline & TABLEAU 4 \\
\hline Flux monétaires & Zone de " luxe " & Zone "verte " & Macro-zone V/L \\
\hline Hôtels & $2500=1000$ & $0200=0$ & 1000 \\
\hline Campings & $0200=0$ & $4150=600$ & 600 \\
\hline Total & 1000 & 600 & 1600 \\
\hline Avant spécialisation & 900 & 500 & 1400 \\
\hline Gains & +100 & +100 & +200 \\
\hline
\end{tabular}

auraient fait le constat suivant illustré au tableau 3.

On traduit alors ces résultats comme suit :

Remarquons tout d'abord qu'au tableau 1 et selon les termes de Ricardo, la zone de « luxe » dispose d'un avantage absolu dans l'exploitation des deux types d'établissements puisqu'ils engendrent tous deux en L un flux monétaire supérieur à celui qu'ils engendrent en V (500 contre 200 pour $\mathrm{H}$; 200 contre 150 pour C). Cependant, au tableau 3, on constate qu'en L, un hôtel engendre 2,5 fois le flux monétaire engendré par un camping alors que dans la zone « verte », ce même rapport n'est que de 1,33 ; ce qui signifie que, comparativement, un hôtel est plus efficient en L qu'en V. On dira que la zone de «luxe » dispose d'un avantage comparatif dans l'exploitation du type de tourisme représenté par les hôtels. Â l'inverse, on constate que si un camping rapporte, en zone de « luxe », 0,4 fois ce que rapporte un hôtel, dans la zone « verte », un camping engendre 0,75 fois le flux monétaire d'un hôtel ; cela signifie que les campings sont comparativement plus efficients dans la zone «verte ». On dira que la zone «verte» dispose d'un avantage comparatif dans l'exploitation du type de tourisme représenté par les campings.

On peut alors se demander si, comme pour l'échange international, les deux zones n'auraient pas intérêt à se spécialiser dans le type de tourisme qu'elles savent exploiter le mieux. Supposons dans notre exemple que, suivant la logique de spécialisation, la zone de « luxe » reçoive les deux hôtels à implanter sur la macro-zone V-L et que la zone « verte » soit, elle, chargée d'exploiter les quatre campings également prévus sur V-L. Les flux monétaires engendrés par les mêmes établissements que précédemment (deux hôtels ; quatre campings) et selon les mêmes moyennes (tableau 1), mais en zones différentes, sont représentés au tableau 4.

En se spécialisant dans l'exploitation d'hôtels, la zone de « luxe » obtient un flux monétaire de 1000 unités monétaires contre 900 avant la spécialisation, soit un gain au plan du flux monétaire de +100 U.M. La zone « verte » obtient le même gain de 100 U.M. en se spécialisant dans le camping, ce qui provoque un gain total de 200 U.M. pour la macro-zone V-L. 


\section{IMPORTANCE DU PRODUIT "IDENTITAIRE "}

\section{COMPORTEMENTS DU CONSOMMATEUR}

Intéressons-nous à présent au « fonctionnement » du client. Nous allons analyser la logique qui pousse le consommateur jusqu'à son choix final vers un produit et tout spécialement vers un produit touristique. Nous verrons alors que la stratégie de développement touristique par la spécialisation infra-régionale est également adaptée à la demande. Rappelons que plusieurs éléments différents interviennent lors de l'élaboration d'un produit touristique ; il s'agit d'une combinaison de deux prestations sans lesquelles il n'y a pas de tourisme possible : transport et hébergement, auxquelles s'ajoute une série de prestations annexes variées. Ces prestations couvrent un large champ d'activités, de la prestation élémentaire comme le petit déjeuner dans un hôtel à l'activité qui constitue le thème majeur du séjour, donc du produit. Le consommateur devra ainsi choisir entre deux produits touristiques celui qui possède les caractéristiques qui constitueront (en quantité et en qualité) la meilleure offre.

Ce choix sera en définitive le résultat d'une série de choix préliminaires concernant l'ensemble des éléments constitutifs du produit qu'il désire. C'est un peu comme si le consommateur réalisait lui-même son produit final en choisissant une combinaison qui regroupe un type de transport, un type d'hébergement, un type d'activité et, pourquoi pas, un type de zone de villégiature. L'exemple suivant donne une idée concrète de ce phénomène : deux habitants d'une même ville, souhaitant passer quelques jours dans la capitale française (Paris), pourront choisir des types de transport différents. L'un utilisera sa voiture, l'autre préférera peut-être utiliser le T.G.V. Le transport est lui-même constitué de plusieurs modes de déplacements. Le simple choix du mode de déplacement constituera plus globalement un choix à l'intérieur du produit touristique final. En effet, pour un même budget initial, le touriste qui utilise son véhicule fera (peut-être) une économie qui lui permettra de choisir un hôtel trois étoiles au lieu d'un deux étoiles. Celui qui préférera le T.G.V. pourra passer deux heures de plus dans un musée. On com- prend bien que le produit final ne sera pas le même pour les deux clients (le choix du transport s'effectue généralement selon des critères de prix, de temps et de confort). On suit le même type de raisonnement en prenant, pour exemple, les différents hébergements offerts. Le produit touristique se définit donc selon les différentes spécificités des services (et biens) qui le constituent. Un consommateur ne choisit donc pas un produit touristique, mais les éléments qui le caractérisent. On ne peut pas demander à quelqu'un de choisir entre une semaine à New York et une semaine à Londres sans lui préciser que l'un des séjours comprend un aller-retour en avion avec pension complète dans un hôtel quatre

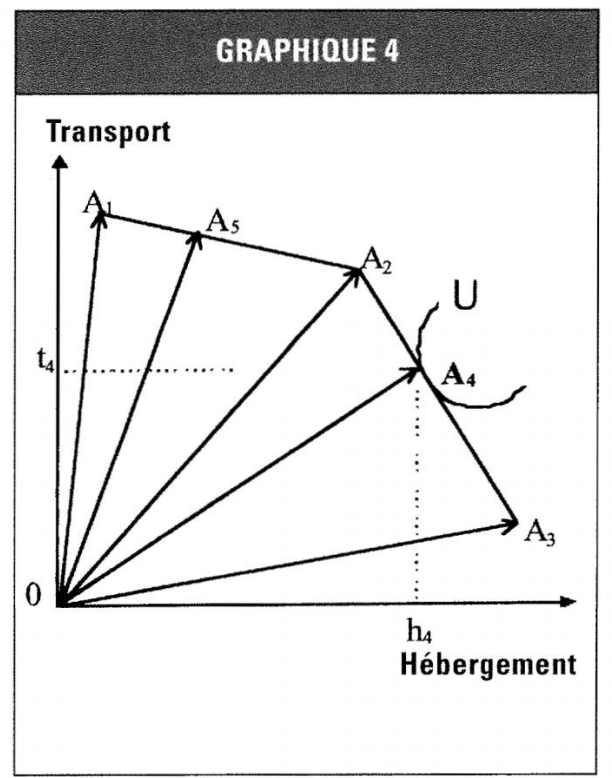

étoiles tandis que l'autre doit s'effectuer en stop avec sac à dos et tente.

Cette manière d'effectuer un choix a fait 1'objet des travaux de Kelvin Lancaster (1971) dans ce que les économistes appellent encore aujourd'hui « la nouvelle théorie du consommateur ». Ce sont les bases de cette théorie qui aident à mieux comprendre comment s'effectue le choix final du consommateur. Cette nouvelle approche se différencie de la théorie traditionnelle essentiellement du fait que les produits ne sont plus les objets directs de l'utilité. En effet, l'utilité ne dépend plus du produit lui-même, mais de ses propriétés, de ses caractéristiques. Les inputs correspondent aux produits, les outputs aux paniers de leurs caractéristiques, l'utilité classe les caractéristiques et donc, indirectement, les produits. De ce fait, un bien apportera, même en consommation de base, plusieurs outputs. Ce bien possédant ses propres caractéristiques indépendamment du consommateur, le choix de ce dernier s'effectuera en fonction de ce qu'il peut lui apporter et non en fonction de ce qu'il est (le produit lui-même).

Ainsi, dans un séjour touristique $\mathrm{S}$ constitué (pour un même budget et vers une même destination) soit d'un transport de première classe et d'un hébergement trois étoiles; soit d'un transport de deuxième classe et d'un hébergement quatre étoiles, Lancaster verra deux produits associés à des vecteurs de satisfaction qui ne diffèrent que par certains éléments les constituant et pour lesquels on peut prendre en compte le choix du consommateur.

Un exemple : sur le graphique 4, parmi les séjours les plus efficients (accessibles au consommateur), le consommateur choisit le séjour x4 (représenté par le vecteur 0A4) dont le type d'hébergement h4 et le type de transport $\mathrm{t} 4$ forment la combinaison de caractéristiques lui apportant la meilleure utilité (un autre consommateur préférera peut-être le séjour x 5 d'une moindre qualité en matière d'hébergement, mais plus appréciable pour ce qui est du transport).

On pourrait conclure cette présentation de la « nouvelle approche du choix du consommateur » de la façon suivante : en définitive, ce n'est pas le choix d'un produit touristique qui doit amener un vacancier à utiliser tel mode de transport et tel type d'hébergement ; ce sont ses préférences de transport et d'hébergement qui l'amèneront à sélectionner ce produit touristique plutôt que cet autre. Il faut donc concevoir des produits qui correspondent aux préférences des consommateurs.

L'hébergement et le transport ne sont évidemment pas les seuls critères que prend en compte le visiteur quand il choisit son produit touristique (I'utilisation de deux éléments seulement n'a ici pour but que de faciliter l'explication du principe de la théorie de Lancaster). Le consommateur choisira en fait tous les éléments constituant le type de tourisme qu'il recherche. Effectivement, si les critères de choix préférentiel du consommateur sont en fait des éléments correspondant à un tourisme culturel (le visiteur recherche un lieu riche en patrimoine historique, musées, visites guidées, coutumes encore bien implantées et respectées, etc.), les sites regroupant ces spécificités seront automa- 
tiquement présents sur la frontière d'efficience du client. Les zones «plage, soleil, farniente » seront éliminées d'emblée en raison de leur faible contenu culturel. À partir de là, le choix subjectif du consommateur se fera (sur la frontière) en fonction de critères auxquels il attribuait jusqu'alors une moindre importance. Deux zones disposant des principaux atouts nécessaires à un tourisme culturel, l'une plus accessible, l'autre plus isolée, feront toutes deux partie de la frontière et constitueront tout de même deux produits touristiques différents puisqu'un consommateur préférera un lieu facile d'accès pour le cas où une obligation l'entraînerait à rentrer momentanément chez lui, tandis qu'un autre préférera s'assurer un certain repos dans une zone plus tranquille car moins visitée (à cause de / grâce à son isolement).

Cette nouvelle théorie semble plus applicable à la réalité bien qu'elle implique que les caractéristiques soient divisibles et additives, ce qui n'est pas toujours le cas. Cependant, il faut reconnaître qu'elle s'adapte très bien au problème que peut se poser le vacancier quant au choix de son site de destination (et de son produit touristique final). En effet, si celui-ci ne faisait aucune différence entre un site et un autre, considérant que tous deux constituent un même produit, le choix se ferait de manière

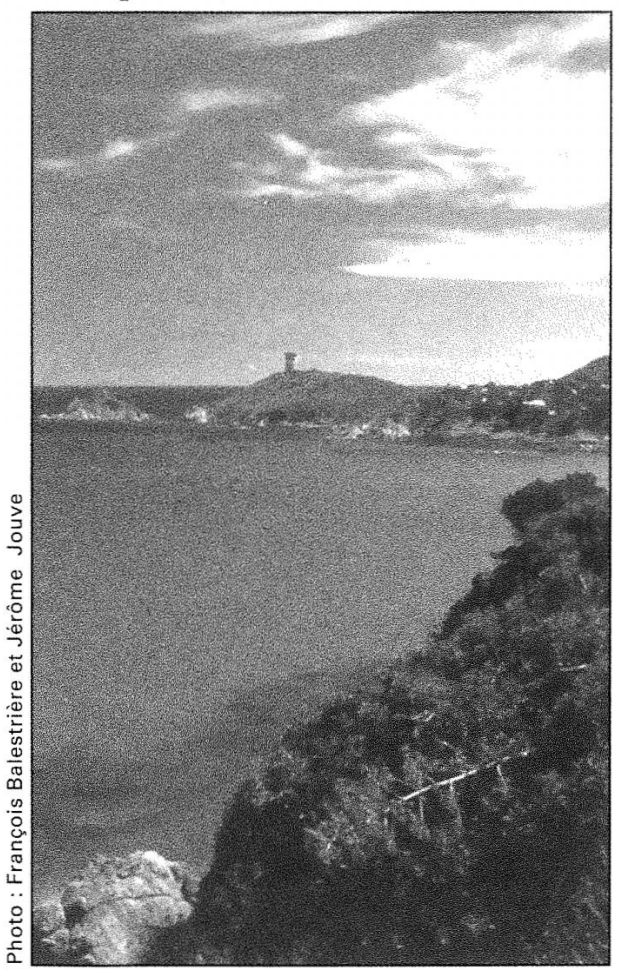

La tour gênoise de Fautea aléatoire et, selon une loi de probabilité, tous les sites d'une même région recevraient chaque année sensiblement un même nombre de visiteurs, ce qui n'est évidemment pas le cas. C'est donc que le consommateur effectue son choix en raison de l'utilité que lui apporteront les différentes caractéristiques constituant une zone (proximité de la plage, musée etc.). La spécialisation par zone facilite le choix du consommateur puisqu'elle fonctionne un peu comme lui. Et peut-être mieux encore : plutôt que de rechercher comme le fait le consommateur à partir de l'idée générale d'un produit final les éléments qui le constitueront, en respectant ses attentes plus précises, éléments correspondant donc à ses goûts de manière plus pointue, la théorie proposée dans cet article veut que la prise en compte des critères d'une zone soit à la base de la réflexion. Ce sont ces éléments de départ qui, étant plus ou moins bien adaptés à tel ou tel produit en particulier, orienteront la zone vers la production du bien final susceptible d'être le plus performant, c'est-à-dire susceptible de correspondre aux attentes du consommateur. Répétons-le, ce n'est donc pas le choix d'un produit touristique qui doit amener un vacancier à utiliser tel mode de transport et tel type d'hébergement, ce sont ses préférences au plan du transport et de l'hébergement qui l'amèneront à sélectionner un produit touristique plutôt qu'un autre. Il faut donc concevoir des produits qui correspondent aux préférences du consommateur.

\section{UNE CONSÉQUENCE PARTICULIÈRE}

L'insularité pourrait être considérée comme un «désavantage » pour ce qui est du produit touristique puisque l'un de ses éléments "le transport » implique une augmentation (négative) de la caractéristique « prix du séjour »; le consommateur d'un produit balnéaire (plage, farniente) considérera peut-être l'offre "Côte d'Azur » plus efficiente que l'offre «Corse» (par exemple) puisque cette dernière produirait une réduction du temps de séjour, causée par le temps de trajet Corse-Continent, et une augmentation du prix du séjour liée au coût de ce même trajet. Cependant, l'insularité peut se transformer en avantage car elle donne une impression plus grande de voyage, de dépaysement, liée justement à la traversée de la Méditerranée et au potentiel culturel de l'île. On pourrait rendre le produit « Corse » au moins aussi efficient que celui « Côte d'Azur », d'une part, en mettant plus en évidence cette caractéristique (culturelle) qui lui est propre et, d'autre part, en réduisant son « désavantage » côté transport. Le Navire à Grande Vitesse (N.G.V.) créé par la « Société Nationale Corse Méditerranée » (S.N.C.M.), en est un parfait exemple puisqu'il permet de diminuer à la fois l'inconvénient financier (moins coûteux que l'avion) et celui de la perte de temps (plus rapide qu'un ferry). Le consommateur préférant le produit « Côte d'Azur » pour une meilleure performance de sa caractéristique «transport » et malgré son désavantage (face à la Corse) sur la caractéristique « dépaysement $»$ remettra son choix en question s'il peut disposer du service (de l'outil) N.G.V. rendant l'écart d'efficience au niveau du transport moins important que celui existant au niveau du dépaysement (favorable à la Corse).

Le professeur Bernard Fustier (1997) montre formellement encore l'intérêt pour une petite économie isolée de « jouer » sur ses particularités. Reprenant le principe du modèle Lancasterien avec :

$\mathrm{I}=\{1 \ldots \mathrm{i} \ldots \mathrm{n}\}$ un ensemble de caractéristiques

et $\mathrm{J}=\{1 \ldots \mathrm{j} \ldots \mathrm{m}\}$ un ensemble de biens concurrents

Il considère (pour une quantité $\mathrm{L}$ de travail) que la quantité zij de caractéristiques i obtenue dans la production du bien $\mathrm{j}$ est réalisée selon une fonction homogène de degré 1 , soit :

$$
\mathrm{zij}=\operatorname{bij}(\mathrm{L} / \mathrm{aj})
$$

bij représente la quantité de caractéristiques i incorporée dans l'unité du bien $\mathrm{j}$;

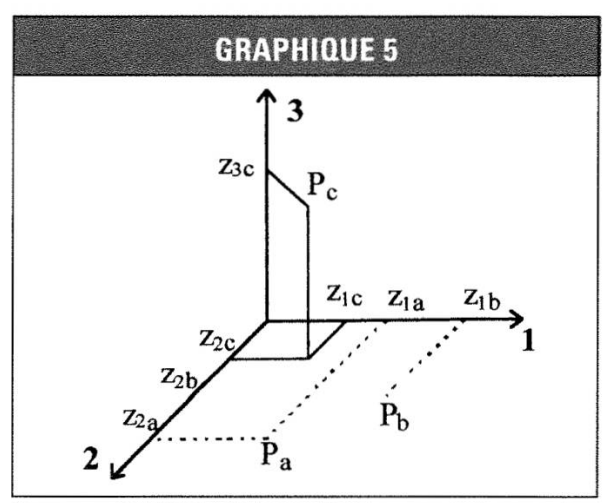

"La diversification des produits par l'intégration de caractéristiques identitaires est source d'efficience pour les régions qui possèdent une forte spécificité socioculturelle." 
aj est la quantité de travail nécessaire à la production d'une unité du bien $\mathrm{j}$.

On note $\mathrm{Pj}$ le faisceau de caractéristiques associé au bien $\mathrm{j}$, soit :

$$
P j=(z i j / i=1 \ldots n)
$$

On définit alors :

- Une « caractéristique identitaire»: « une caractéristique spécifique au patrimoine culturel d'un territoire est dite identitaire ».

- Un « bien identitaire » : « un bien est dit identitaire si, et seulement si, le faisceau de caractéristiques qui lui est associé intègre au moins une caractéristique identitaire ».

- Une relation de «domination » : «le faisceau $\mathrm{Pj}$ domine le faisceau $\mathrm{Ph}$ si, et seulement si : $\ll \mathrm{i}, \mathrm{i}=1 \ldots \mathrm{n}$; on a $: \mathrm{zij} \geq$ zih avec une inégalité stricte pour au moins une caractéristique $\mathrm{i} \gg$.
- La notion «d'efficience »: « un bien est dit efficient si, et seulement si, le faisceau de caractéristiques qui lui est associé n'est dominé par aucun autre faisceau $»$.

Le professeur Fustier fait remarquer que dans la mesure où une caractéristique identitaire est spécifique, un bien identitaire possède au moins une caractéristique que ne possèdent pas les biens concurrents : un bien identitaire est donc forcément un bien efficient. Considérons trois entreprises localisées en des régions différentes $a, b$, c et produisant des biens concurrents avec la même quantité de travail. Les caractéristiques 1 et 2 sont des caractéristiques ordinaires en ce sens qu'elles rentrent habituellement dans la composition du type de produit considéré. En revanche, 3 est une caractéristique identitaire inhérente à la région c. Dans cette dernière région, la productivité du travail est plus faible que dans les deux autres (le besoin unitaire en travail dans $c$ est plus grand que dans les deux autres régions). Si l'on admet que les coefficients bij ne sont pas touchés par le lieu de localisation de l'entreprise, alors la position relative des faisceaux de caractéristiques, compte tenu de la fonction de production utilisée, dépend uniquement de la disparité spatiale de la productivité du travail. Dans l'espace à deux dimensions des caractéristiques ordinaires ( 1 et 2), on voit (sur le graphique 5) que, contrairement aux biens produits dans les deux autres régions, le bien produit en $\mathrm{c}$ n'est pas efficient. Mais dans l'espace à trois dimensions (prenant en considération la caractéristique 3 inhérente à la région c), aucun faisceau de caractéristiques n'est dominé par un autre faisceau : tous les biens sont efficients.

\section{CONCLUSION}

Appuyer son développement sur ses propres spécificités (forme de développement identitaire), même si elles sont moins performantes que celles de la concurrence (pour celles qui ne sont pas identitaires),

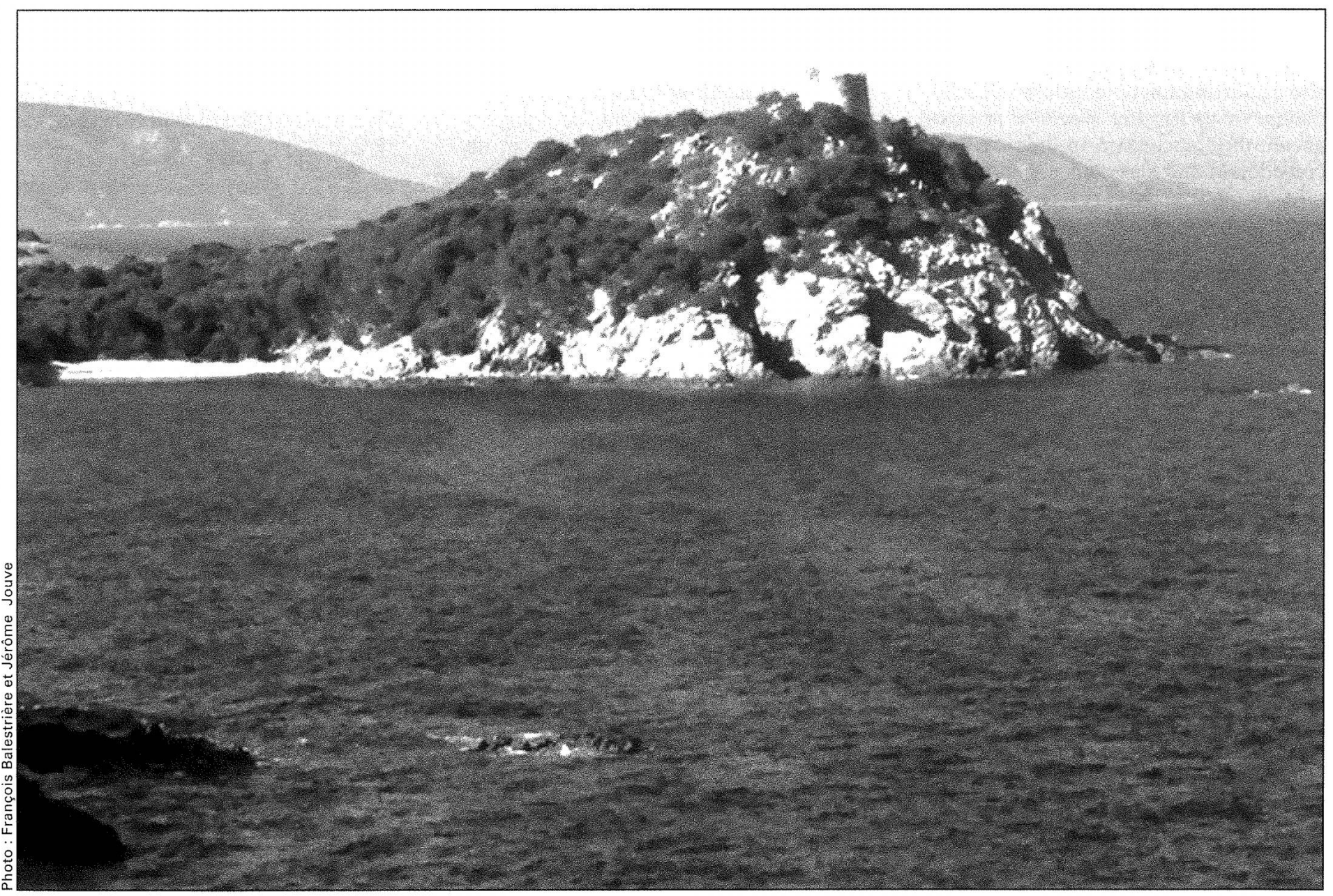

La tour de Sponsaglia 
permet souvent d'augmenter l'efficience globale d'une région touristique, en augmentant son avantage comparatif. La spécialisation nécessite évidemment une forme de coopération inter-zone pour être effective (et donc efficace), chaque décideur devant accepter de se spécialiser. Cette entente aura alors pour intérêt de :

- Générer des externalités liées, sinon à la proximité, en tous cas à la présence sur un même territoire d'une gamme de produits variés (disponibles sur des entités relativement voisines).

- Assurer la durabilité du développement puisque le secteur «non-basic » (résidentiel) répond à une demande de l'activité de base sinon stable, en tous cas plus étalée dans le temps (sur l'année).

- Être compatible avec la mise en valeur de produits identitaires (y compris culturels) dans la mesure où certaines zones seront spécialisées dans un tourisme dit identitaire.

Finalement, la spécialisation infra-régionale repose sur l'idée de développement endogène puisque les zones se spécialisent dans le produit le mieux adapté à leurs caractéristiques intrinsèques, pratiques et besoins de la population locale inclus (notamment dans le cadre du tourisme identitaire), sans exclure la vision réaliste de la théorie de la base puisque le moteur de la croissance régionale repose sur les exportations (dépenses des touristes) et nécessite l'existence et l'intégration d'un secteur résidentiel fort, capable de répondre à la demande des touristes, se comportant ainsi en fournisseur de la base.

Denis Serra effectue actuellement un stage postdoctoral au CIFORT (Centre international de formation et de recherche en tourisme) de l'UQAM, sous la tutelle du professeur Louis Jolin; dirigé par le professeur Bernard Fustier, il a soutenu sa thèse de doctorat ès sciences économiques à l'Université de Corse (France).

Un comité de lecture a lu et accepté ce texte

D'autres photos de la Corse sont disponibles sur le site www.acorsica.com

\section{NOTES}

1 On peut difficilement dissocier « les chutes » de « Niagara » et inversement elles font partie du patrimoine et de l'identité même de la région. Ainsi, ce phénomène naturel est une caractéristique « identitaire ». De même, tout produit touristique intégrant (entre autres activités) la visite des chutes sera pour Niagara un produit identitaire puisque basé sur une particularité qui lui est propre. Notons alors qu'une caractéristique identitaire ne sera pas forcément liée au paysage exceptionnel d'une zone, mais pourra également provenir du patrimoine historique de cette dernière (comme les plages de Normandie, théâtre du débarquement des Alliés durant la Seconde Guerre mondiale) ou plus simplement d'une spécificité gastronomique. La mise en valeur d'une telle caractéristique confère alors à la zone qui en bénéficie une situation de monopole, un avantage certain sur ses concurrents directs.

2 Certaines îles françaises de la Caraïbe et de l'Océan Indien bénéficient d'un avantage considérable du fait de l'alignement des tarifs publics, notamment de l'électricité, sur ceux de la métropole ; il en résulte, pour ces îles favorisées, de possibles économies d'échelle qui viennent remettre en cause la compétitivité globale des produits touristiques.

3 Effectivement, le prix à payer pour acheminer une marchandise d'un point initial aux cinquante (premiers) kilomètres environnants, est plus important que celui qu'occasionnerait une augmentation de cinquante kilomètres sur un marché se situant à deux cents kilomètres du point initial (de fabrication)

4 Dans la théorie de la base, seules les activités exportatrices permettent à l'espace considéré de se développer (de sortir d'une situation de stagnation économique, d'un système clos), elles constituent le secteur de base. On parle alors, en opposition, des secteurs résiduels qui n'exportent pas. Toutefois, nombre d'auteurs préfèrent parler de secteurs résidentiels (terme peut-être moins péjoratif) puisqu'ils permettent de subvenir aux besoins des populations locales.

\section{BIBLIOGRAPHIE}

Brémond, Janine, et Alain Gélédan (1984), Dictionnaire des théories et des mécanismes économiques, Hatier.

Catin, Maurice (1994), «Externalités » et «Économie d'agglomération», dans Auray, Bailly, Derycke, Huriot, Encyclopédie d'économie spatiale, Economica.

Crusol, J., P. Hein, et F. Vellas (1988), L'enjeu des petites économies insulaires, Economica.

Durand, H., P. Gouirand, et J. Spindler (1994), Économie et politique du tourisme, Librairie droit jurisprudence (Systèmes).

Faini, R. (1988), « Problèmes de développement spécifiques aux économies insulaires », dans Crusol, Hein, Vellas, L'enjeu des petites économies insulaires, Economica.

Fustier, Bernard (1995), « Position économique des régions insulaires par rapport à la norme Européenne : le poids de l'isolement ", Revue d'Économie Régionale et Urbaine, $\mathrm{n}^{\circ} 1$.

Fustier, Bernard (1997), « Une stratégie de développement fondée sur la production de biens identitaires ", Actes du colloque international d'Arles/France, Économie des Régions Méditerranéennes et Développement Durable.

Jayet H., J.P. Puig, et J.F. Thisse (1996), «Enjeux économiques de l'organisation du territoire », Revue d'économie politique.

Lacour, Claude (1973), Aménagement du territoire et développement régional, Dalloz.
Lancaster, Kelvin J. (1971), A New Approach to Consumer Theory, Columbia University Press.

Polèse, Mario (1994), Économie urbaine et régionale : logique spatiale de mutations économiques, Economica.

Serra, Denis (2000), Tourisme et développement régional : proposition d'une stratégie de spécialisation infra-régionale adaptée aux spécificités des petites économies isolées, thèse de doctorat ès sciences économiques de l'Université de Corse.

Serra, Denis (1998), « Coopérer par la spécialisation: pour une organisation du tourisme Méditerranéen ", dans les Actes du colloque international de Split/Croatie, Cooperation and Development in the Mediterranean, Première rencontre des économistes Méditerranéens.

Serra, Denis (1997), « Reflexion sur l'organisation du tourisme insulaire fondée sur l'analyse de l'offre et de la demande ", Actes du colloque international d'Arles/France, Économie des Régions Méditerranéennes et Développement Durable.

Serra, Denis (1994), Développement touristique corse : vers une spécialisation micro-régionale, mémoire de maîtrise ès sciences économiques, Université de Corse.

Vellas, François (1985), Économie politique du tourisme international, Economica.

Vellas, François (1992), Le tourisme, Economica. 